\section{Tabaco, álcool e outras drogas entre adolescentes em Pelotas, Rio Grande do Sul, Brasil: uma perspectiva de gênero}

\author{
Tobacco, alcohol, and drug use by teenagers \\ in Pelotas, Rio Grande do Sul State, Brazil: \\ a gender approach
}

Rogério Lessa Horta 1,2,3

Bernardo Lessa Horta 4

Ricardo Tavares Pinheiro 5

Blanca Morales 1

Marlene Neves Strey ${ }^{3}$
1 Prontamente Clínica da Família, Porto Alegre, Brasil. 2 Universidade do Vale do Rio dos Sinos, São Leopoldo, Brasil.

3 Pontifícia Universidade Católica do Rio Grande do Sul, Porto Alegre, Brasil.

${ }_{4}$ Universidade Federal de Pelotas, Pelotas, Brasil. 5 Universidade Católica de Pelotas, Pelotas, Brasil.

Correspondência

R. L. Horta

Prontamente Clínica da Família.

Rua Domingos Rubbo 348 , apto. 903, Porto Alegre, RS 91040-000, Brasil.

rogeriohorta@hotmail.com

\section{Abstract}

This study assesses the relationship between gender and use of psychoactive substances (alcohol, nicotine, and illicit drugs) by teenagers. In 2002, a cross-sectional study was carried out in the urban area of Pelotas, southern Brazil. Multi-stage sampling was used to obtain a sample of adolescents, 15 to 18 years of age. Subjects were interviewed using a self-applied confidential questionnaire. Smoking was more prevalent among girls, while alcohol consumption in the previous month was more common among boys. Meanwhile, the proportion of adolescents that reported drug use in the previous month was unrelated to gender. Higher cigarette consumption by girls suggests an increase in smoking by women in the future, which highlights the need for a special focus on this area.

Adolescent; Smoking; Gender Identity; Alcoholic Beverages

\section{Introdução}

A sociedade contemporânea está imersa nas revoluções da informação e da tecnologia, que afetam, diariamente, a vida dos indivíduos e dos grupos, invadindo o mundo perceptivo e os sistemas de crenças, com novos padrões de comportamento e com intenso apelo ao consumo 1 .

Os novos tempos trazem também novas configurações familiares e a transformação no exercício dos papéis de gênero. Mulheres e homens disputam espaços no mercado de trabalho e as estereotipias e papéis definidos em outros tempos perdem espaço 2,3,4.

A indústria das substâncias psicoativas ocupa os espaços de difusão da oferta e da sedução ao consumo e está atenta aos movimentos sociais, como os fenômenos da inclusão das mulheres no mercado de trabalho ou o da expansão da mídia eletrônica 5.

Nesse sentido, é fundamental que se identifiquem, sempre de modo ágil, populações com tendência ao incremento no consumo de álcool, tabaco e outras drogas, procurando identificar, também, fatores determinantes ou associados à mudança de comportamentos. Isso pode melhor orientar ações nos campos da prevenção e do tratamento dos problemas decorrentes do uso de substâncias psicoativas, hoje largamente deficientes $6,7,8$

A população adolescente merece ser acompanhada com especial atenção nesse sentido. 
Esse grupo populacional já apresentava, no levantamento de base domiciliar do Brasil, em 2001, indicadores de prevalência discretamente distintos dos demais grupos populacionais e tem despertado o interesse de inúmeros grupos de pesquisa neste campo 9,10,11,12,13.

A informação de que homens fumam mais, bebem mais e consomem mais drogas que as mulheres 14 pode estar entre os elementos em transformação nos cenários atuais no campo da saúde 15. As mulheres caracterizam grupo social de relevância para estudos sobre o uso de substâncias psicoativas por haver provável expansão dos indicadores de consumo e dos problemas decorrentes do consumo de substâncias psicoativas nesta população 5, por estarem cada vez mais presentes em estudos que têm por base populações de sujeitos em tratamento por problemas com substâncias psicoativas 16,17 e pela interface que estes campos de estudos mantêm com áreas como a dos estudos em saúde materno-infantil 18,19,20.

Este artigo apresenta dados levantados a partir do estudo Prevalência dos Comportamentos em Saúde em Adolescentes na Cidade de Pelotas, comparando as informações prestadas por meninas e por meninos, com o objetivo de examinar a relação entre gênero e consumo de substâncias psicoativas (bebidas alcoólicas, tabaco e drogas ilícitas) entre adolescentes.

\section{Método}

Em estudo realizado no Município de Pelotas, Rio Grande do Sul, Brasil, no ano de 2002, a equipe de pesquisas do Programa de Pós-graduação em Saúde e Comportamento da Universidade Católica de Pelotas (UCPel) entrevistou 960 adolescentes, entre 15 e 18 anos de idade, residentes na zona urbana do município. A amostragem foi feita em múltiplos estágios, a partir dos 448 setores censitários da zona urbana. Desses setores, 90 foram sorteados sistematicamente para inclusão no estudo. A seguir, selecionou-se, aleatoriamente, um quarteirão de cada setor e foi feito o sorteio de uma esquina deste quarteirão para ser o ponto inicial com base no qual 86 residências foram visitadas de forma sistemática. Quando necessário, casas de outros quarteirões foram incluídas, escolhidas de forma padronizada a fim de completar o número de 86 residências. No total, 7.740 domicílios foram visitados pela equipe de pesquisa. Em cada domicílio sorteado foram entrevistados todos os adolescentes com idade entre 15 e 18 anos completos, após ser obtido o consentimento por escrito dos pais ou de outra pessoa adulta responsável.
Não foi possível realizar a entrevista com 79 $(7,6 \%)$ dos 1.039 adolescentes localizados por recusa na obtenção da autorização dos pais ou responsáveis, dos próprios adolescentes em responderem ao questionário ou por não ter sido encontrados em casa após três revisitas. O procedimento de revisitas foi empregado para reduzir a possibilidade de ocorrência de viés de seleção.

Os sujeitos responderam a um questionário auto-aplicado, no qual se buscavam informações sobre comportamentos em saúde. Foram selecionados, para o exame neste artigo, os desfechos:

- Em relação ao tabaco: (i) uso na vida ou experimentação: consumo, pelo menos uma vez, ao longo da vida, e (ii) uso continuado ou recente: consumo uma vez por semana, pelo menos, no mês que antecedeu as entrevistas.

- Em relação a bebidas alcoólicas: foi solicitado que se informasse ter havido ou não consumo de bebidas alcoólicas no mês que antecedeu as entrevistas.

- Em relação a drogas ilícitas (maconha, cocaína e/ou solventes): (i) uso na vida ou experimentação: consumo, pelo menos uma vez, ao longo da vida, e (ii) uso recente: consumo no mês que antecedeu as entrevistas.

Foram tomadas como variáveis independentes a idade, o sexo, a escolaridade, o comparecimento à escola no presente, a ocorrência de reprovação escolar e a classe social.

A variável classe social foi definida com base na classificação preconizada pela Associação Brasileira de Institutos de Pesquisa de Mercado (ABIPEME), que define classe A como a de maior acesso a bens de consumo e serviços, e a classe $\mathrm{E}$ como a de menor acesso.

Os questionários foram digitados com o programa Epi Info (Centes for Disease Control and Prevention, Atlanta, Estados Unidos) e foi feita uma checagem automática dos dados no momento da digitação com o uso do programa Check do Epi Info, a fim de identificar e corrigir inconsistências na digitação.

Foram realizadas análises univariadas e bivariadas. Adotou-se o teste de qui-quadrado $\left(\chi^{2}\right)$ com nível de significância de $5 \%$ nos testes de diferenças de proporções.

\section{Considerações éticas}

O questionário era auto-aplicado, sem dados de identificação pessoal, e era depositado por quem o respondera, logo após a entrevista, em uma urna lacrada, o que garantia o sigilo das informações. $\mathrm{O}$ 
projeto foi aprovado pelo Comitê de Ética em Pesquisa da UCPel e pela Fundação de Amparo à Pesquisa do Estado do Rio Grande do Sul (FAPERGS).

\section{Resultados}

Na amostra estudada, $36 \%$ dos adolescentes pertenciam a famílias da classe social A ou B, enquanto que $26 \%$ eram da classe D ou E. Quanto à escolaridade do adolescente, 83,4\% dos entrevistados estavam freqüentando a escola e $58 \%$ tinham nove anos ou mais de escolaridade. Finalmente, cerca de dois em cada três adolescentes foram reprovados pelo menos uma vez na escola.

Em relação ao consumo de cigarros, identificou-se relato de uso continuado (uma vez por semana ou mais, no último mês) em 157 sujeitos, ou $16,6 \%$ dos respondentes. Para uso na vida, foram obtidas 408 respostas afirmativas, ou $43 \%$ dos adolescentes que responderam a esta questão, afirmaram já ter experimentado cigarro, pelo menos uma vez.
A análise segundo o sexo mostrou que 19,5\% das meninas entrevistadas afirmaram consumir cigarros uma vez por semana ou mais no mês que antecedeu as entrevistas, enquanto $13 \%$ dos meninos afirmaram fazê-lo $(\mathrm{p}<0,05)$. A prevalência de tabagismo foi 1,51 vez (IC95\%: 1,142,00) maior entre as meninas.

O uso de tabaco também foi mais freqüente entre adolescentes com mais de 17 anos, de classe social D ou E e com menor escolaridade (Tabela 1).

Tendo como referência adolescentes de classe social A, os grupos de classes C e D/E apresentaram, respectivamente, razões de prevalência para uso recente de cigarros de 1,22 (IC95\%: 0,911,64) e 1,67 (IC95\%: 1,16-2,42). Foram encontradas razões de prevalência, para o mesmo desfecho, de 1,88 (IC95\%: 1,64-2,94) e 1,50 (IC95\%: 1,12-2,01) entre adolescentes com quatro anos de estudo ou menos e entre aqueles com 5 a 6 anos, tendo como referência o grupo de jovens com nove anos de estudo ou mais. O grupo que referiu reprovação escolar apresentava prevalência 2,76 (IC95\%: 1,64-4,65) vezes maior de consu-

Prevalência de tabagismo entre adolescentes de acordo com sexo, idade, classe social e escolaridade. Pelotas, Rio Grande do Sul, Brasil, 2002.

\begin{tabular}{|c|c|c|c|}
\hline Variáveis & $\mathbf{n}$ & $\begin{array}{c}\text { Consumo de cigarros no } \\
\text { último mês (\%) }\end{array}$ & $\begin{array}{c}\text { Razão de prevalência } \\
\text { (IC95\%) }\end{array}$ \\
\hline Sexo & & $p<0,05$ & \\
\hline Feminino & 497 & 19,5 & $1,51(1,14-2,00)$ \\
\hline Masculino & 463 & 13,0 & Referência \\
\hline Idade (anos) & & $p<0,01$ & \\
\hline 15 & 227 & 11,0 & Referência \\
\hline 16 & 271 & 13,3 & $1,21(0,85-1,74)$ \\
\hline 17 & 221 & 20,8 & $1,89(1,32-2,74)$ \\
\hline 18 & 241 & 20,8 & $1,89(1,32-2,74)$ \\
\hline Classe social & & $p<0,05$ & \\
\hline $\mathrm{A} / \mathrm{B}$ & 346 & 13,0 & Referência \\
\hline $\mathrm{C}$ & 366 & 15,9 & $1,22(0,91-1,64)$ \\
\hline $\mathrm{D} / \mathrm{E}$ & 248 & 21,8 & $1,67(1,16-2,42)$ \\
\hline Adolescente está estudando & & $p>0,05$ & \\
\hline Sim & 801 & 15,4 & Referência \\
\hline Não & 159 & 21,4 & $1,39(0,98-1,98)$ \\
\hline Escolaridade do adolescente (anos) & & $p<0,05$ & \\
\hline$\leq 4$ & 52 & 25,0 & $1,88(1,20-2,94)$ \\
\hline $5-8$ & 351 & 19,9 & $1,50(1,12-2,01)$ \\
\hline$\geq 9$ & 557 & 13,3 & Referência \\
\hline Ocorrência de reprovação escolar & & $p<0,05$ & \\
\hline Sim & 589 & 21,7 & $2,76(1,64-4,65)$ \\
\hline Não & 368 & 7,9 & Referência \\
\hline
\end{tabular}

$N=960$ 
mo de cigarros no último mês que o grupo que não registrou reprovações.

A Tabela 2 mostra que a maior freqüência de uso de fumo entre as meninas se mantém para experimentação na vida e para uso continuado. A relação entre uso na vida e uso continuado mostrou-se equivalente entre os sexos, numa razão de 2,6:1, indicando que de cada 2,6 jovens que experimentam cigarros, um permanece em uso continuado, pelo menos dentro da faixa etária estudada.

Entre os meninos encontrou-se freqüência mais elevada de consumo do álcool no último mês $(\mathrm{p}<0,01)$, como mostra a Tabela 3 . Além disso, o consumo de bebidas alcoólicas esteve associado com idade e com reprovação escolar. O consumo de bebidas alcoólicas no mês das entrevistas, tendo como referência o grupo de adolescentes de 18 anos de idade, foi referido 1,12 (IC95\%: 0,92-1,36) vez por aqueles com 17 anos, 0,87 (IC95\%: 0,71-1,06) vez pelos de 16 anos e 0,75 pelos de 15 anos (IC95\%: 0,69-0,94) $(\mathrm{p}=0,01)$. Foi encontrada, também, razão de prevalência 1,16 (IC96\%: 1,00-1,34) vez maior para o grupo de adolescentes que apresentou reprovação escolar ( $\mathrm{p}<0,05)$.

Em relação ao uso de drogas ilícitas, o uso recente de maconha, cocaína ou solventes foi referido por $9 \%$ dos adolescentes, enquanto o uso na vida foi referido por $14,9 \%$ da população estudada.

O uso na vida das drogas ilícitas estudadas não apresentou diferença significativa quanto ao sexo, nem quanto à idade ou classe social. Esse desfecho esteve associado apenas às variáveis escolaridade do adolescente, ter freqüentado a escola e à ocorrência de reprovação escolar. Entre adolescentes, respectivamente, com quatro anos de estudo ou menos e entre 5 e 8 anos de estudo completos, o uso de drogas ilícitas na vida foi referido 2,08 vezes (IC95\%: 1,23-3,54) e 1,06 vez (IC95\%: 0,77-1,46), se comparados ao grupo de sujeitos com nove anos de estudos ou mais $(\mathrm{p}=0,01)$. Perguntados se haviam freqüentado a escola no último ano, o grupo que não esteve na escola apresentou razão de prevalência para este desfecho 1,65 (IC95\%: 1,08-2,50) vez maior que o grupo que o fez $(p=0,02)$. Em relação à ocorrência de reprovação escolar, o grupo que referiu este fato teve razão de prevalência para uso de drogas ilícitas na vida 1,82 (IC95\%: 1,24-2,67) vez maior que o grupo sem reprovações $(p=0,01)$, como mostra a Tabela 4.

O uso de drogas ilícitas no mês que antecedeu as entrevistas não apresentou diferença significativa apenas quanto à classe social. Esse desfecho esteve associado a todas as demais variáveis: sexo, idade, escolaridade, ter freqüentado a escola e ocorrência de reprovação escolar. A razão de prevalência para meninas, tendo como referência o grupo de adolescentes do sexo masculino, foi de 0,58 (IC95\%: 0,38-0,91) $(\mathrm{p}<0,05)$. Quanto à idade, tendo como referência o grupo de jovens de 18 anos, os grupos de 15, 16 e 17 anos tiveram razões de prevalência para este desfecho, respectivamente, de 0,68 (IC95\%: 0,37-1,23), 0,65 (IC95\%: 0,36-1,15) e 1,54 (IC95\%: $0,87-2,72)(\mathrm{p}<0,05)$.

Entre adolescentes com, respectivamente, quatro anos de estudo ou menos e 5 a 8 anos de estudo completos, o uso de drogas ilícitas no mês foi referido 3,25 vezes (IC95\%: 1,59-6,62) e 1,92 vez (IC95\%: 1,17-3,15), se comparados ao grupo de sujeitos com nove anos de estudos ou mais $(\mathrm{p}<0,05)$. Perguntados se haviam freqüentado a escola no último ano, o grupo que não esteve na escola apresentou razão de prevalência 1,71 (IC95\%: 1,04-2,80) vez maior que o grupo que o fez $(\mathrm{p}<0,05)$. Em relação à ocorrência de reprovação escolar, o grupo que referiu este fato teve razão de prevalência para uso de cocaína, maconha e/ou solventes no mês 2,26 (IC95\%: 1,39$3,66)$ vezes maior que o grupo sem reprovações, como mostra a Tabela 4 .

Tabela 2

Relação entre uso continuado de tabaco e experimentação na vida, segundo o sexo.

Adolescentes de 15 a 18 anos, Pelotas, Rio Grande do Sul, Brasil.

\begin{tabular}{lccc}
\hline Sexo & $\begin{array}{c}\text { Fumo na vida } \\
\text { (A) }\end{array}$ & $\begin{array}{c}\text { Uso continuado (uma vez/semana } \\
\text { ou mais, no último mês) (B) }\end{array}$ & $\begin{array}{c}\text { Relação experimentação/ } \\
\text { uso continuado (A:B) }\end{array}$ \\
\hline Feminino & 249 & 97 & $2,6: 1$ \\
Masculino & 159 & 60 & $2,6: 1$ \\
Total & 408 & 157 & $2,6: 1$ \\
P & $<0,001$ & $<0,001$ & \\
\hline
\end{tabular}


Prevalência de ingestão de bebidas alcoólicas no último mês entre adolescentes de acordo com sexo, idade, classe social e escolaridade. Pelotas, Rio Grande do Sul, Brasil, 2002.

\begin{tabular}{|c|c|c|c|}
\hline Variáveis & n & $\begin{array}{c}\text { Ingestão de bebida alcoólica } \\
\text { no último mês (\%) }\end{array}$ & $\begin{array}{c}\text { Razão de prevalência } \\
\text { (IC95\%) }\end{array}$ \\
\hline Sexo & & $p<0,01$ & \\
\hline Feminino & 497 & 37,9 & Referência \\
\hline Masculino & 463 & 49,0 & $1,29(1,14-1,47)$ \\
\hline Idade (anos) & & $p=0,01$ & \\
\hline 15 & 227 & 34,7 & $0,75(0,59-0,94)$ \\
\hline 16 & 271 & 40,4 & $0,87(0,71-1,06)$ \\
\hline 17 & 221 & 52,1 & $1,12(0,92-1,36)$ \\
\hline 18 & 241 & 46,4 & Referência \\
\hline Classe social & & $p>0,05$ & \\
\hline$A / B$ & 346 & 47,5 & Referência \\
\hline C & 366 & 42,6 & $0,90(0,77-1,04)$ \\
\hline $\mathrm{D} / \mathrm{E}$ & 248 & 38,2 & $0,80(0,67-0,96)$ \\
\hline Adolescente está estudando & & $p>0,05$ & \\
\hline Sim & 801 & 43,1 & Referência \\
\hline Não & 159 & 44,0 & $1,02(0,87-1,20)$ \\
\hline Escolaridade do adolescente (anos) & & $p>0,05$ & \\
\hline$\leq 4$ & 52 & 38,8 & $0,89(0,60-1,31)$ \\
\hline $5-8$ & 351 & 43,4 & $0,99(0,85-1,16)$ \\
\hline$\geq 9$ & 557 & 43,6 & Referência \\
\hline Ocorrência de reprovação escolar & & $p<0,05$ & \\
\hline Sim & 589 & 45,7 & $1,16(1,00-1,34)$ \\
\hline Não & 368 & 39,5 & Referência \\
\hline
\end{tabular}

$N=960$.

\section{Discussão}

Os dados deste estudo restringem-se à população urbana de um município de médio porte (com mais de 200 mil habitantes), da Região Sul do país. Outros recortes poderiam determinar resultados distintos destes.

As diferenças entre os sexos, aqui mapeadas, concordam com achados do estudo domiciliar do Brasil. Em 2001, Carlini et al. 14 encontraram uma estimativa de uso na vida de tabaco que somava $46,3 \%$ entre os homens e $36,3 \%$ entre as mulheres. Porém, entre jovens, de 12 a 17 anos, havia um discreto predomínio entre as meninas, com 16,2\% delas afirmando já ter consumido cigarros contra $15,2 \%$ dos meninos. Nas dezoito maiores cidades da Região Sul do país, aqueles autores encontraram que $19,3 \%$ das meninas e $18,2 \%$ dos meninos já haviam consumido cigarros. Resultados semelhantes já apareciam em estudo anterior 21, também no Município de Pelotas.
O consumo de substâncias psicoativas, bem como uma série de outros comportamentos humanos, parece associar-se a questões de gênero, entre outras variáveis. $\mathrm{O}$ sexo de quem respondia às entrevistas esteve associado a distintos padrões de contato com cada um dos grupos de substâncias e isto pode estar relacionado a condições históricas e sociais determinadas pelo modo como se constroem os conceitos de masculino e feminino 22.

Pode ser que as meninas apenas tenham os primeiros contatos com o tabaco mais cedo que os meninos ou pode estar começando a ser percebido um movimento que levaria ao estabelecimento de padrões de consumo de tabaco maiores para mulheres que para homens em gerações futuras.

Qualquer dos dois cenários (início precoce ou aumento global da prevalência), que não são excludentes entre si, implica preocupação imediata do ponto de vista de saúde pública, mais especificamente, do ponto de vista da saúde das mulheres. 
Prevalência de uso de drogas ilícitas na vida e no último mês, segundo sexo, idade, classe social e escolaridade. Pelotas, Rio Grande do Sul, Brasil, 2002.

\begin{tabular}{|c|c|c|c|c|c|}
\hline \multirow[t]{2}{*}{ Variáveis } & \multirow[t]{2}{*}{$\mathbf{n}$} & \multicolumn{4}{|c|}{ Uso de drogas ilícitas } \\
\hline & & Na vida & RP (IC95\%) & No último mês & RP (IC95\%) \\
\hline Sexo & & $p>0,05$ & & $p<0,05$ & \\
\hline Feminino & 497 & 13,9 & $0,96(0,66-1,40)$ & 6,4 & $0,58(0,38-0,91)$ \\
\hline Masculino & 463 & 14,6 & Referência & 11,0 & Referência \\
\hline Idade (anos) & & $p>0,05$ & & $p<0,05$ & \\
\hline 15 & 227 & 11,6 & $0,64(0,39-1,05)$ & 6,2 & $0,68(0,37-1,23)$ \\
\hline 16 & 271 & 12,0 & $0,66(0,45-0,98)$ & 5,9 & $0,65(0,36-1,15)$ \\
\hline 17 & 221 & 15,6 & $0,84(0,58-1,20)$ & 14,0 & $1,54(0,87-2,72)$ \\
\hline 18 & 241 & 18,2 & Referência & 9,1 & Referência \\
\hline Classe social & & $p>0,05$ & & $p>0,05$ & \\
\hline$A / B$ & 346 & 16,1 & Referência & 7,2 & Referência \\
\hline C & 366 & 12,6 & $0,77(0,56-1,07)$ & 9,3 & $1,29(0,68-2,44)$ \\
\hline $\mathrm{D} / \mathrm{E}$ & 248 & 14,1 & $0,86(0,55-1,34)$ & 9,7 & $1,34(0,69-2,58)$ \\
\hline Escolaridade do adolescente (anos) & & $p=0,01$ & & $p<0,05$ & \\
\hline$\leq 4$ & 52 & 28,6 & $2,08(1,23-3,54)$ & 19,2 & $3,25(1,59-6,62)$ \\
\hline $5-8$ & 351 & 14,0 & $1,06(0,77-1,46)$ & 11,4 & $1,92(1,17-3,15)$ \\
\hline$\geq 9$ & 557 & 13,1 & Referência & 5,9 & Referência \\
\hline Freqüentou a escola no último ano & & $p=0,02$ & & $p<0,05$ & \\
\hline Sim & 801 & 12,7 & Referência & 7,6 & Referência \\
\hline Não & 159 & 21,2 & $1,65(1,08-2,50)$ & 13,2 & $1,71(1,04-2,80)$ \\
\hline Ocorrência de reprovação escolar & & $p=0,01$ & & $p<0,05$ & \\
\hline Sim & 589 & 17,3 & $1,82(1,24-2,67)$ & 11,0 & $2,26(1,39-3,66)$ \\
\hline Não & 368 & 9,3 & Referência & 4,9 & Referência \\
\hline
\end{tabular}

$N=960$.

Em 1998, Costa-e-Silva \& Koifman 5 apontavam que quatro fatores sócio-demográficos em larga expansão na América Latina tornaram esta parte do mundo especialmente atrativa para a indústria multinacional do tabaco: o incremento progressivo da população jovem, o aumento de um padrão de vida urbano, maior acesso à educação e o acesso das mulheres ao mercado de trabalho. Os quatro parâmetros reunidos apontariam mulheres jovens, urbanas e com maior escolaridade como a população-alvo preferencial da indústria tabagista. O artigo cita que a indústria do tabaco se volta para esse mercado a partir da década de 50.

Mesmo que a tendência de crescimento neste momento seja mais restrita ao fumo, sabe-se que o uso de substâncias psicoativas costuma produzir um efeito multiplicador, em que o consumo de uma substância aumenta o risco de consumo de outras. Mulheres fumantes, por exemplo, têm até duas vezes mais chances de consumirem bebidas alcoólicas que as não-fumantes 15 . Esse efeito não parece evidente nos dados aqui relatados, uma vez que os indicadores de prevalência de uso de álcool e de uso de maconha, cocaína ou solventes não acompanham a mesma relação de gênero que o consumo de tabaco.

O estudo de Carlini et al. 14, de base domiciliar realizado nas 107 maiores cidades do Brasil, usou como indicadores do comportamento dos sujeitos em relação ao álcool as variáveis "uso na vida" e "uso regular" (este último definido como beber pelo menos três dias na semana). Neste estudo, trabalhou-se com a ocorrência de consumo no mês que antecedeu as entrevistas. Isso faz com que os dados não possam ser comparados com precisão, mas destacam-se a proximidade entre os valores encontrados por Carlini et al. para uso na vida $(52,2 \%$ entre meninos de 12 a 17 anos e $44,7 \%$ entre meninas da mesma idade) e os que foram aqui descritos. Os dois estudos concordam ao indicar maior ocorrência de consumo de álcool entre adolescentes do sexo masculino.

Para a variável consumo de drogas ilícitas, este estudo e aquele realizado no país mostraram resultados distintos. Considerando-se que não foram percebidos erros metodológicos, supõe-se que a população jovem do Município de Pelo- 
tas apresente uma tendência peculiar, superior à média nacional à experimentação de cocaína, maconha ou solventes. Esses dados podem indicar, também, que a população de Pelotas informe mais sobre drogas ilícitas que populações de outras localidades. O município é sede de um centro de pesquisas epidemiológicas com tradição na realização de estudos de base domiciliar e a população pode, de algum modo, estar mais convencida quanto à sua segurança em relação às informações prestadas.

Do ponto de vista da saúde das populações, os dados aqui apresentados parecem prenunciar um alto preço a ser pago no futuro, uma vez que as complicações decorrentes do uso de tabaco, álcool e ilícitas resultam em ocorrências letais, mas de incidência retardada em relação às mudanças dos padrões de consumo, especialmente o tabaco 23 .

Seria lamentável que as lutas pela democratização, emancipação das mulheres e de outros segmentos sociais não se fizessem acompanhar de maior crítica e aprofundamento das reflexões sobre o que nos põe em risco. Cerca de $90 \%$ dos casos de câncer de pulmão no mundo são atribuíveis ao tabagismo 24.

A ruptura de alguns mecanismos sociais de inibição de comportamentos danosos ou de risco, sem a necessária realocação de mecanismos de proteção pode ser recorrente nas revoluções: a explosão revolucionária desconstitui a autoridade e seus referentes, que se recolocam apenas à medida que os eventos demandem novos delimitadores e à medida que se constitua consenso social para tanto ${ }^{25}$. A revolução feminista e a aparente associação entre a emancipação das mulheres e o aumento da prevalência de consumo de cigarros entre as jovens parecem refletir essa idéia. Os achados deste estudo não confirmam plenamente a ocorrência dessa expansão do consumo de cigarro por mulheres, mas não ajudam a desconstituir a preocupação neste sentido. Sanahuja-Yll 26 examina as diversas formas de organização, expressão e mobilização das mulheres ao longo da luta por sua emancipação. A autora aponta diversas entidades, teorias e princípios estabelecidos desde os primórdios da transformação de seu papel na sociedade, mas não faz referência a estudos que relacionem este processo emancipatório a indicadores de consumo de substâncias psicoativas. Esses dados podem servir, ainda, como estímulo para que os movimentos feministas e suas lideranças, bem como outros movimentos e setores da sociedade, assumam sua responsabilidade e aportem alternativas não tradicionais para o enfrentamento dos danos produzidos pelo uso de substâncias psicoativas.
O estabelecimento de comportamentos como o tabagismo e o consumo de outras substâncias psicoativas é fenômeno complexo e não poderia ser explicado em associação a uma única variável ou a um único fenômeno. Devem estar envolvidos no debate representantes da comunidade científica, das equipes de saúde e também das mulheres e de seus movimentos e entidades, além de um grande número de outros setores, como a mídia, empresas e profissionais da comunicação, da educação, da saúde, do direito, das ciências sociais, empresas e empresários, além de gestores públicos.

Políticas públicas, setoriais ou institucionais, poderiam desestimular, conter ou contribuir para a reversão desses comportamentos.

A eficácia das políticas públicas, postas em xeque à medida que dados novos apontam mais freqüentemente para incremento do consumo de álcool, tabaco e drogas, deve ser o ponto central deste debate, como já tem sido apontado por outros autores 6,7,27,28,29,30,31.

Dos dados aqui apresentados, destacam-se ainda a preocupante associação entre um menor desempenho escolar e a ocorrência de fumo e uso de drogas. O uso de drogas apareceu associado também à inexistência de vínculo com a escola e a maior ocorrência de reprovações escolares. Parece preocupante que populações com menor acesso à educação formal estejam mais expostas aos riscos decorrentes do consumo daquelas substâncias. Esses dados devem ser considerados no planejamento de políticas voltadas à população mais jovem. Nesta amostra, em Pelotas, constata-se a ocorrência elevada de consumo de drogas ilícitas entre adolescentes que não estão mais na escola ou que somam, no máximo, quatro anos de estudo. A exclusão da educação formal elimina a escola como uma rota de acesso às políticas de prevenção e atenção a usuários de substâncias psicoativas.

Os dados deste estudo parecem indicar que o uso de drogas ilícitas, álcool e tabaco variam segundo o sexo e a idade da população adolescente na cidade de Pelotas. As meninas estão fumando mais que os meninos e estes consomem mais álcool que as meninas e parecem estar fazendo uso continuado de drogas ilícitas em maior percentual do que as meninas, apesar dos dados relativos à ocorrência de experimentação de drogas ilícitas ao longo da vida não ter dependido do sexo de quem respondia. Jovens de 17 e 18 anos, independente do sexo, consomem mais álcool, tabaco e drogas ilícitas que aqueles com idades entre 15 e 16 anos.

Classe social, escolaridade, vínculo com a escola e a ocorrência de reprovações escolares estiveram mais associadas ao consumo de tabaco 
e drogas ilícitas, estando o consumo de drogas ilícitas intimamente associado a prejuízo no desempenho escolar e da permanência na escola, e o consumo de tabaco tendo incidido mais entre populações com menor escolaridade.

Pela complexidade do tema, parece recomendável que se amplie a discussão com o envolvimento de movimentos sociais e setores diversos da sociedade civil e mídia, além de gestores públicos.

A replicação de estudos de base populacional, em pontos distintos do território nacional e com certa periodicidade, poderia aumentar a capacidade de monitoramento desses indicadores, subsidiando, com maior qualidade, o debate em torno do tema.

\section{Resumo}

Este estudo teve o objetivo de examinar a relação entre gênero e utilização de substâncias psicoativas entre adolescentes (consumo de bebidas alcoólicas, fumo e uso de drogas ilícitas). Em 2002 foi realizado um estudo transversal na área urbana de Pelotas, extremo-sul do Brasil. Foi empregada amostragem em múltiplos estágios para se obter uma amostra de adolescentes entre 15 e 18 anos de idade. As entrevistas foram realizadas com questionário auto-aplicado. Tabagismo foi mais prevalente entre as meninas, enquanto o consumo de bebida alcoólica no mês anterior às entrevistas foi maior entre os meninos. Por outro lado, a proporção de adolescentes que relataram uso de drogas ilícitas no mês que antecedeu as entrevistas não esteve relacionada ao gênero. O maior consumo de tabaco entre meninas que entre meninos pode indicar risco de expansão do consumo desta substância por mulheres de outras faixas etárias em gerações futuras, o que justifica preocupação e esforços específicos.

Adolescente; Tabagismo; Identidade de Gênero; Bebidas Alcoólicas

\section{Colaboradores}

R. L. Horta participou da análise de dados e coordenou a discussão dos resultados e a organização e redação final deste artigo. B. L. Horta planejou e coordenou a coleta e a análise dos dados e participou da redação final do artigo. R. T. Pinheiro participou da elaboração do instrumento, do delineamento do estudo, acompanhou a coleta e a análise de dados e contribuiu para a discussão e elaboração deste artigo. M. N. Strey colaborou na discussão e análise dos dados e na redação final do artigo. B. Morales participou na revisão da literatura, discussão e redação do artigo.

\section{Agradecimentos}

$\mathrm{O}$ projeto original obteve financiamento junto à Fundação de Amparo à Pesquisa do Estado do Rio Grande do Sul. 


\section{Referências}

1. Pearce WB. Novos modelos e metáforas comunicacionais: a passagem da teoria à prática, do objetivismo ao construcionismo social e da representação à reflexividade. In: Schnitman DF, organizador. Novos paradigmas, cultura e subjetividade. Porto Alegre: Editora Artes Médicas; 1996. p. 172-83.

2. Hennigen I, Guareschi NMF. A paternidade na contemporaneidade: um estudo de mídia sob a perspectiva dos estudos culturais. Psicol Soc 2002; 14:44-68.

3. Grzybowski LS. Famílias monoparentais: reflexo da pós-modernidade? In: Guareschi P, Pizzinato A, Kruger LL, Macedo MMK, organizadores. Psicologia em questão reflexões sobre a contemporaneidade. Porto Alegre: Edipucrs; 2003. p. 113-22.

4. Instituto Brasileiro de Geografia e Estatística. Perfil das mulheres responsáveis pelos domicílios no Brasil - 2000. Rio de Janeiro: Instituto Brasileiro de Geografia e Estatística; 2002. (Estudos e Pesquisas - Informação Demográfica e Sócio-econômica, 8).

5. Costa-e-Silva VL, Koifman S. Smoking in Latin America: a major public health problem. Cad Saúde Pública 1998; 14 Suppl 3:99-108.

6. Uchtenhagen A. How effective is substance abuse prevention? World Psychiatry 2005; 4:33.

7. Selby P, Vaccarino F. Substance abuse prevention: practical strategies for psychiatrists in the $21^{\text {st }}$ century. World Psychiatry 2005: 4:32-3.

8. El-Guebaly N. Don't drink and drive: the successful message of Mothers agaInst Drunk Driving (MADD). World Psychiatry 2005; 4:35-6.

9. Giusti J, Sañudo A, Scivoletto S. Differences in the pattern of drug use between male and female adolescents in treatment. Rev Bras Psiquiatr 2002; 24:80-2.

10. Silva VA, Aguiar AS, Felix F, Rebello GP, Andrade RC, Mattos HF. Brazilian study on substance misuse in adolescents: associated factors and adherence to treatment. Rev Bras Psiquiatr 2003; 25:133-8.

11. Soldera MA, Dalgalarrondo P, Corrêa Filho HR, Silva CAM. Uso pesado de álcool por estudantes dos ensinos fundamental e médio de escolas centrais e periféricas de Campinas (SP): prevalência e fatores associados. Rev Bras Psiquiatr 2004; 26:174-9.

12. Dalgalarrondo P, Soldera MA, Corrêa Filho HR, Silva CAM. Religião e uso de drogas por adolescentes. Rev Bras Psiquiatr 2004; 26:82-90.

13. Dalgalarrondo P, Soldera MA, Corrêa Filho HR, Silva CAM. Jovens pentecostais e espíritas em comparação a católicos: uso de álcool e drogas e saúde mental. J Bras Psiquiatr 2005; 54:182-90.

14. Carlini EA, Galduróz JCF, Noto AR, Nappo SA, organizadores. I levantamento domiciliar sobre o uso de drogas psicotrópicas no Brasil. São Paulo: Centro Brasileiro de Informações sobre Drogas Psicotrópicas; 2002.
15. Kroeff LR, Mengue SS, Schmidt MI, Duncan BB, Favaretto ALF, Nucci LB. Correlates of smoking in pregnant women in six Brazilian cities. Rev Saúde Pública 2004; 38:261-7.

16. Ribeiro MS, Alves MJM, Guirro UBP, Baldi BG. Alcoolismo: a influência do reconhecimento da comorbidade na adesão de pacientes ao programa terapêutico. J Bras Psiquiatr 2004; 53:124-32.

17. Souza RS, Siqueira MM. O processo de enfermagem na assistência a pacientes com dependência de álcool. J Bras Psiquiatr 2005; 54:228-33.

18. Halal IS, Victora CG, Barros FC. Determining factors related to smoking and its abandonment during pregnancy in an urban locality in Southern Brazil. Rev Saúde Pública 1993; 27:105-12.

19. Muller JS, Antunes M, Behle I, Teixeira L, Zielinsky P. Acute effects of maternal smoking on fetal-placental-maternal system hemodynamics. Arq Bras Cardiol 2002; 78:152-5.

20. Leopercio W, Gigliotti A. Smoking and its peculiarities during pregnancy: a critical review. J Bras Pneumol 2004; 30:176-85.

21. Horta BL, Calheiros P, Pinheiro RT, Tomasi E, Amaral KC. Tabagismo em adolescentes de área urbana da região sul do Brasil. Rev Saúde Pública 2001; 35:159-64.

22. Strey MN, organizador. Construções e perspectivas em gênero. São Leopoldo: Editora Unisinos; 2000.

23. Nakamura MU, Alexandre SM, Santos JFK, Souza E, Sass N, Beck APA, et al. Obstetric and perinatal effects of active and/or passive smoking during pregnancy. São Paulo Med J 2004: 122:94-8.

24. Wunsch-Filho V, Bonffetta P, Colin D. Familial cancer aggregation and the risk of lung cancer. São Paulo Med J 2002; 120:38-44.

25. Hobsbawm E. l'ere des revolutions. Brussels: Éditions Complexe; 1988.

26. Sanahuja-Yll ME. Cuerpos sexuados, objetos y prehistoria. Madrid: Ediciones Cátedra; 2002.

27. Marques ACPR, Campana A, Gigliotti AP, Lourenço MTC, Ferreira MP, Laranjeira R. Consenso sobre o tratamento da dependência de nicotina. Rev Bras Psiquiatr 2001; 23:200-14.

28. El-Guebaly N. Addictions' research in developing countries: adjusting abounding questions to limited resources. Rev Bras Psiquiatr 2005; 27:7-8.

29. Medina-Mora ME. Prevention of substance abuse: a brief overview. World Psychiatry 2005; 4:25-30.

30. Clajus TEJ, Queiroz MS. Prevenção contra o uso e o abuso de drogas: abordagens em debate. J Bras Psiquiatr 2004; 53:90-9.

31. Poznyak V. The role of psychiatrists in prevention of substance use and dependence beyond clinical practice. World Psychiatry 2005; 4:31-2.

Recebido em 20/Dez/2005

Versão final reapresentada em 07/Ago/2006

Aprovado em 20/Out/2006 